
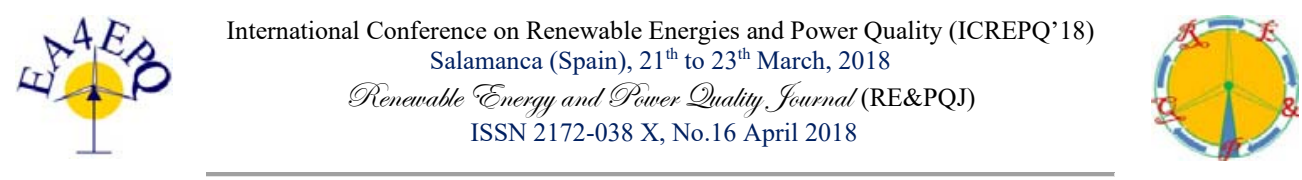

\title{
Microgrid controller standardization - principles and implementation
}

\author{
G. Joos ${ }^{1}$ and J. Reilly ${ }^{2}$ \\ ${ }^{1}$ Department of Electrical and Computer Engineering \\ Montreal, QC, Canada \\ Phone:+ 1514398 7118, e-mail: geza.joos@mcgill.ca \\ ${ }^{2}$ Reilly Associates \\ New Jersey, USA
}

\begin{abstract}
Microgrids are being successfully promoted and deployed as a means to integrate distributed energy resources into distribution systems and manage the combined generation and load as a single entity. This paper demonstrates, by means of successful standardization efforts, that despite the wide range of possible implementations of microgrids and their control system, there are core functions that define the microgrid and exploit the benefits of implementing a microgrid. It presents the standards that define a minimal set of core functions, develops the functional specification of the control system and proposes test approaches and metrics. These core functions allow a basic microgrid control system to be specified and tested for conformance to the proposed standards.
\end{abstract}

\section{Key words}

Microgrid control system, Distributed Energy Resources, Interconnection, Integration, Distribution System, Standards.

\section{Introduction}

For the purposes of the discussion in this paper, a microgrid is defined as a group of interconnected loads and distributed energy resources (DER), with clearly defined electrical boundaries, that acts as a single controllable entity with respect to the grid and can connect and disconnect from the grid to enable operation in both grid-connected or island modes. The control system includes a number of functions that define the microgrid as a system that manages itself, operates autonomously, and connects to and disconnects from the main distribution grid for the exchange of power and the supply of ancillary services, energy management functions, and other ancillary functions as required. DER, as defined in this paper, include distributed generation (conventional and renewable, wind and solar), energy storage (battery), and controllable loads (for demand response).

Microgrids are being deployed in increasing numbers in different applications and contexts across distribution systems [1], [2], [3], [4]. There are a number of incentives for deploying microgrids, including the interconnection, integration and management of a number of DER, the need for increased reliability and resilience of the supply of electrical power and the possibility of islanded operation in the event of the inability of the distribution grid to supply the load [2], [5], [6], [7], [8], [12].

The purpose of standardization is to facilitate the wide acceptance and deployment of a given technology. IEEE Std 1547 specifies the interconnection requirements for individual DER [9]. This standard is being revised in response to the increasing penetration of DER and the need for DER to actively support the grid to maintain its reliability and integrity. The revised version recognizes the increasing impact of individual DER on distribution grids and the enhanced flexibility and the ancillary services they can provide [8], [10]. In a parallel effort, standardization of microgrid control systems has been carried out to facilitate the deployment of microgrids, irrespective of the topology, the power and control system and the application [3]. Standard development includes standards recently sponsored by the IEEE Standards Association, namely IEEE P2030.7 and IEEE P2030.8.

A major task in the development of standards for microgrid controllers is defining generic or core functions for the control of microgrid assets, including DER, and of switching and regulating devices under the control of the microgrid control system. The aim is to provide a uniform baseline for the design, configuration and operation of microgrids, and allow interoperability of the offerings from different vendors.

The process of defining core functions for microgrid control systems is the major contribution of this paper. It develops the guiding principles for the functional specification of core functions, characterizes these functions, develops approaches for testing them, and discussed the available testing environments, including the use of real time simulators. It addresses metrics that need to be met by microgrid control systems for conformance. 


\section{Distributed Energy Resources Integration}

\section{A. DER aggregation - DERMS, VPP and microgrids}

Distributed Energy Resources (DER) are being deployed in increasing numbers in distribution systems. DER consists, among others, of generators using renewable energy resources, namely solar and wind, alternative fuels (bio-gas and bio-mass), conventional fuels (natural gas and diesel fuel), and energy efficient generation technologies such as combined heat and power (CHP). In the case of renewable energy resources that are variable and intermittent, generators using these resources are nondispatchable, and their output power cannot be controlled, other than being partially or fully curtailed. Battery energy storage systems, also a DER, can be associated with these generators to allow regulating their output power.

An advantage of making use of and integrating DER is the ability to feed local loads without making use of the transmission infrastructure. This allows enhanced reliability and resilience of the distribution system and the possibility of feeding loads during faults and outages on the transmission and distribution grids.

When the penetration of DER is low (10\% or less), DER is used essentially to provide real power and has limited impact on the grid. DER based on renewable energy resources are typically operated at their maximum output power level, set by the maximum power point tracking mechanism, to maximize the return on the investment. For higher penetration levels, DER may have an impact on the grid. For enhanced flexibility in the management of DER, they can be aggregated using systems such as Distributed Energy Resources Management Systems (DERMS), Virtual Power Plants (VPP) or microgrids, Fig. 1.

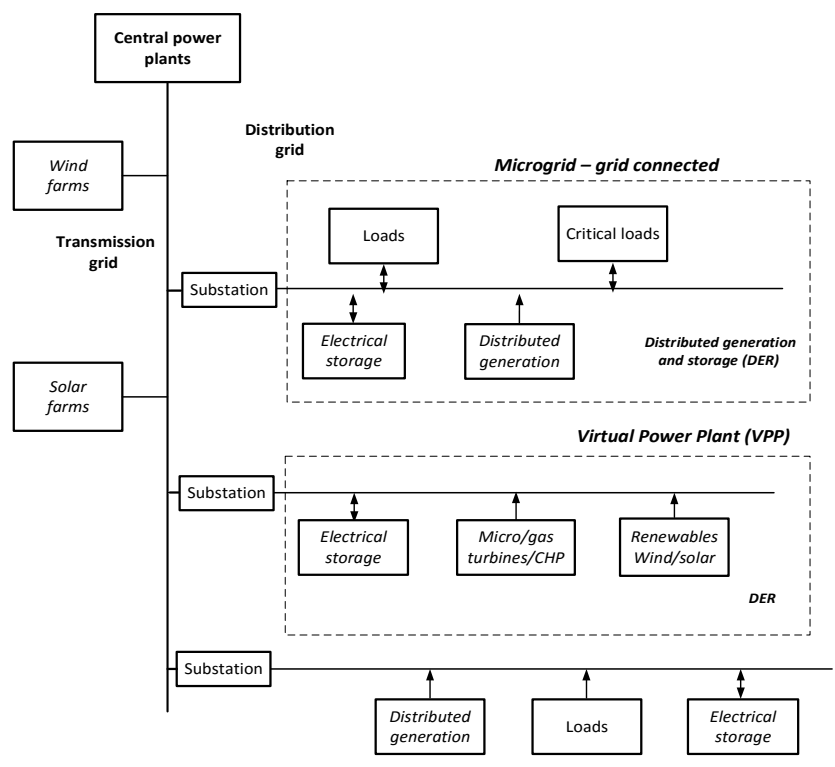

Fig. 1. Integration of DER in distribution systems.
The interconnection of individual DER is dictated by utility grid codes or by standards, such as the IEEE Std 1547. These typically specify grid operating ranges for voltage and frequency under steady state and transient conditions for which the DER needs to remain connected.

\section{B. Microgrids - a tool for aggregation of DER and loads}

Microgrids are an efficient solution for aggregating generation, storage and loads into a single entity that can serve a number of purposes, from the optimization of resources to serve loads within a specified boundary to setting the power flow across the point of interconnection with the distribution utility, participating in markets, and islanding for resiliency.

A microgrid is an aggregation of DER and loads. It consists of a group of interconnected loads and DER with clearly defined electrical boundaries that acts as a single controllable entity with respect to the grid and can connect and disconnect from the grid to enable it to operate in both grid-connected or island modes. The discussion in this paper assumes that the microgrid has a single point of interconnection with the distribution grid. The general structure of the microgrid and its relation to the broader distribution and transmission grids is shown in Fig. 2.

The control system requirements for aggregation include:

1) Energy management function - for local management of DER, particularly variability and intermittency. From a power and energy perspective, this can be done either by means of storage or by load management (load curtailment and demand response). There needs to be an energy management system that dispatches the assets to supply the load and meet contractual agreements at the point of connection to the grid.

2) Function overseeing the transition from grid connected mode to islanded or standalone mode and back - for connect/disconnect, black start capability, system restoration services; supplying local critical loads in the event of the failure of the distribution grid, features that improve the reliability, security and resilience of the electric power supply to the loads within the microgrid. This requires that the microgrid can disconnect and operate in islanded mode.

3) Function managing the interactions with the grid - for power exchanges (real and reactive power) and DMS interaction; DSO and TSO benefits and control. One of the important features and benefits of creating a microgrid is to locally manage DER and loads and present to the DSO a defined net power consumed or fed back into the grid. The recent interest of the TSO to monitor and control DER is directly associated with the impact that aggregated DER can have on the transmission system, particularly the loading on transmission lines and on transmission system capacity. Aggregating DER within a microgrid would facilitate the ability of the TSO to manage the transmission grid. Other functions include the provision of ancillary services to the distribution grid; supply or real and reactive power (voltage support), frequency regulation and others. 


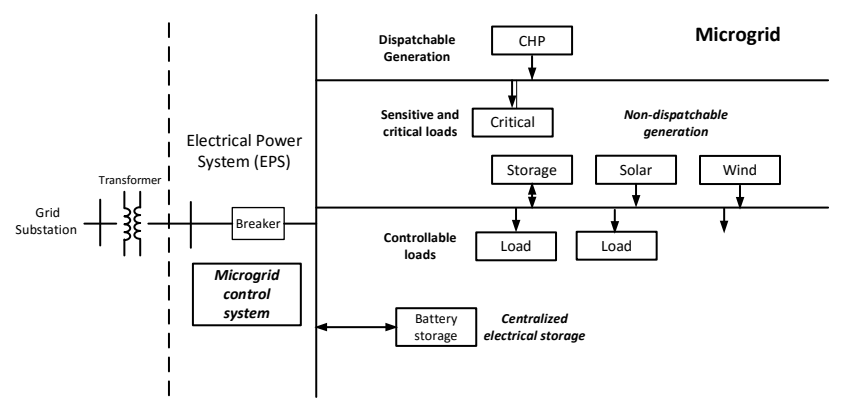

Fig. 2. Microgrid structure and components.

\section{Control System Standardization}

\section{A. Standardization - IEEE P2030.7 and P2030.8}

The IEEE Standards Association has issued Project Authorization Requests for the following:

1) P2030.7 - Standard for the Specification of Microgrid Controllers - "The reason for establishing a standard for the Microgrid Energy Management System (MEMS) is to enable interoperability of the different controllers and components needed to operate the MEMS through cohesive and platform-independent interfaces. This approach will allow for flexibility and customization of components and control algorithms to be deployed without sacrificing or limiting potential functionality."

2) P2030.8 - Standard for the Testing of Microgrid Controllers - "The reason for establishing a standard for testing microgrid controllers, in the context of enabling interoperability of the different controllers and components needed to operate the controller through cohesive and platform-independent interfaces, is to establish standardized testing procedures. The standardization focuses on testing functional requirements."

\section{B. Microgrid control system functions}

The control system manages all aspects of the microgrid operation at the point of connection to the distribution grid, in steady state and under transient conditions. Under steady state operation, the control system dispatches the microgrid assets, including DER units and interface and switching devices. Under transient conditions, the control system is responsible for ensuring the smooth connection and disconnection from the distribution grid. Functions used to control a microgrid are described in Fig. 3.

The functions cover the different levels of operation, from the faster functions (typically in the ms range), the device level functions, associated with the control of the assets, including the DER, to the slower functions (in the min range), the grid interactive control, associated with the interaction with the grid. The intermediate levels deal with the local area control and the supervisory control. The representation is a generic set of functions and the boundaries between blocks can vary from one microgrid control system implementation to another. In addition, since these functions are conceptual functions, there are many possible hardware and software implementations.

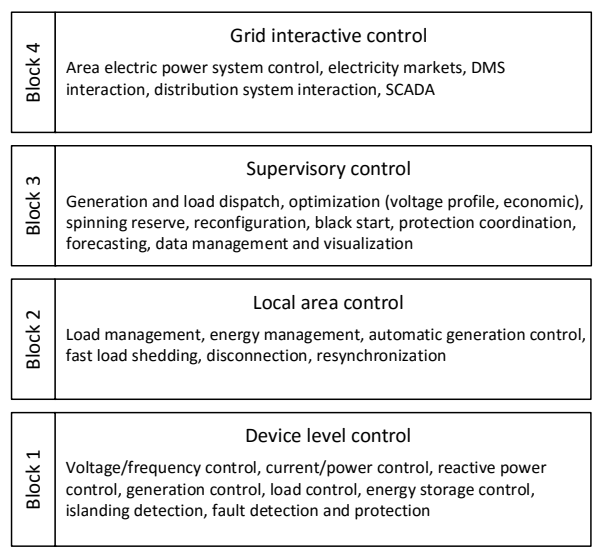

Fig. 3. Microgrid control system functions.

\section{C. $\quad$ Control system core functions - P2030.7}

The two core functions identified as being essential for all microgrids are the following:

1) The dispatch function - It computes and distributes the set-points for generation and storage, including DER units and loads (controllable and curtailable) in grid-connected and islanded modes, under steady state and transient conditions, including disconnection from and reconnection to the grid.

2) The transition function - It defines the operations required to implement the transition from gridconnected to islanded modes, including disconnection from the distribution grid and resynchronization to the grid.

The core functions are made up of functions selected from the blocks of Fig. 3. Because of the nature of the core functions, their role and position in the microgrid control system structure are between the lower and higher-level functions as illustrated in Fig. 4.

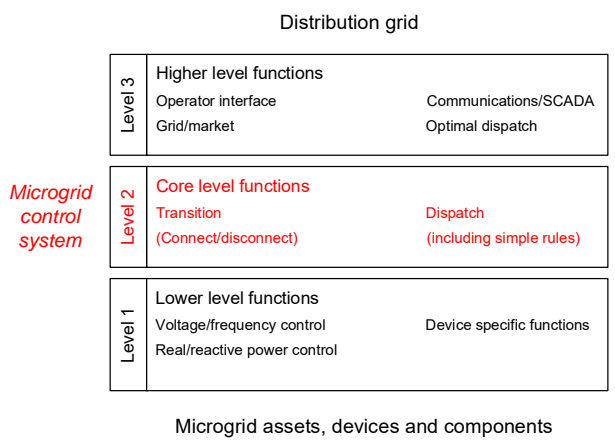

Fig. 4. Microgrid control system core functions. 


\section{Functional specification of core functions - P2030.7}

The functional description and specification of the core functions is summarized below:

1) The dispatch function - It maximizes the use of the assets, including the DER, and ensures that the operation of the microgrid meets the requirements, both for the internal operation and as seen from the point of interconnection to the distribution system. A dispatch order is a set of commands sent to the microgrid assets, devices and components; the commands may be simple rules, or be based on the optimization of predetermined operating modes of the microgrid, including the minimization of the cost of electricity, while serving the required loads; the operating rules may be different in grid connected and islanded modes; under steady state conditions, the dispatch function executes its commands at regular intervals (typically $15 \mathrm{~min}$ or less); during transitions, see below, it executes emergency dispatch orders, on a interrupt basis, to ensure that transitions take place in an orderly manner, dispatching assets and curtailing load.

2) The transition function - It manages transitions and operates in the following four situations: (a) unplanned islanding, resulting from a loss of distribution grid power; (b) planned islanding, resulting from a request from the distribution grid operator to disconnect; (c) reconnection / resynchronization; (d) black start as required in the islanding process. Disconnection from the grid can be carried out without interruption or require a shut-down and restart; this can be in the form of a black start using one of the microgrid assets, either a dispatchable generator or a storage device; the transition function provides the signal to switch the dispatch function from one mode to another, the dispatch function then being responsible for reconfiguring the control system functions during the transitions and as a function of the new mode of operation.

\section{Conformance Testing of Core Function}

A. Approach to the testing of core functions - P2030.8

The purpose of this standard for testing is to establish the minimum requirements for the interoperability between the offerings of different vendors and facilitate the development and deployment of cohesive and platform independent interfaces. A standardized set of testing procedures can facilitate the wide adoption of a standardized microgrid functional specification.

The proposed testing approach for the microgrid control system includes the following elements and steps:

1) Defining test scenarios - Scenarios allow testing the core functions under well-defined and representative conditions; they allow testing of individual core functions or the combination of the two core functions, as in the case of transitions, where the transition function initiates an emergency dispatch order, particularly in the case of an unplanned islanding event. These scenarios assume a generic configuration.

2) Defining performance metrics - These need to consider existing applicable standards related to electric distribution systems, applicable distribution grid requirements and grid codes, and relevant and applicable instrumentation and measurement techniques.

3) Defining the testing environment - This can range from a fully numerical/software environment to a fully hardware (full scale) installation, see Section 5 below. An accepted and readily implementable environment is realtime simulation with a Hardware in the Loop (HIL) approach for testing all or elements of the microgrid control system.

\section{B. Metrics $-P 2030.8$}

In the testing procedures, based on the scenarios used to test the core functions, the variables measured are voltage and current. The derived quantities are frequency, real and reactive power, and power quality related indices (voltage sags and swells, flicker). These are measured at the point of interconnection (POI) to the grid given that the standard only deals with the steady state and transient response of voltage, frequency, and power exchanges at the POI. In islanded mode, other points may be selected.

The typical shape of the allowable operating ranges of voltage and frequency requirements at the POI are depicted in Figures 5 and 6 . These are the same type of plots that apply to any generator or DER connected to a distribution grid. The actual voltage and frequency levels and the interval over which they apply are set either by the interconnection requirements with the DSO, or the applicable grid codes, or set by any applicable or accepted standard in this jurisdiction.

Islanded microgrids can operate under relaxed voltage and frequency constraints, if the equipment is designed accordingly. The islanded constraints are unique to each microgrid.

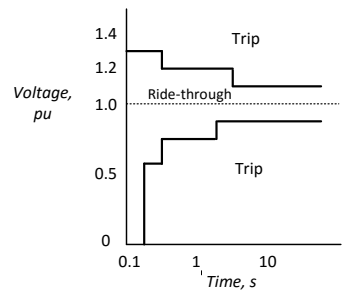

Fig. 5. Generic operational voltage ranges - grid-connected mode.

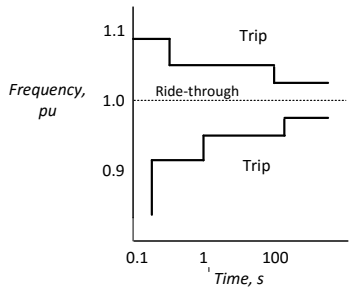

Fig. 6. Generic operational frequency ranges - grid-connected mode. 


\section{Conformance testing}

The standard states that compliance of the microgrid control system with the standard shall be tested in accordance to the test scenarios specified and metrics agreed upon. The test shall be carried out by the vendor jointly with the utility or entity to which the microgrid is connected, and that has issued the interconnection requirements and agreements or the system specification.

\section{Test Facilities and Types of Test Beds}

A number of known and tested approaches, implemented in various forms and in a number of different contexts in electrical power conversion system testing and validation, have been used for testing controllers to verify and tune operation to meet desired functionalities. The more recent approaches use Real Time Simulators (RTS).

\section{A. Controller Hardware in the Loop (C-HIL)}

Controller Hardware-in-the-Loop C-HIL) testing is a common testing methodology which has been used for decades to test complex control and protection systems. It offers a wide range of test coverage with the possibility of automating tests to accelerate the performance assessment of the device under test.

1) Non-Real-Time Controller HIL - This testing methodology uses non-real-time software simulations and slow communication protocols to communicate with the Microgrid Control System (MGCS). This approach only allows for testing slow dispatch scenarios handled by the MGCS. It may be used as a first step in controller prototyping to evaluate the implementation of dispatch rules in the controller software.

2) Real-Time Controller C-HIL - Real-Time C-HIL simulations, Fig. 7, requires that the power components, and/or their controllers, and the rest of the system be simulated in real-time. This requires more powerful computing hardware than is used for pure software simulations. With CHIL, all the high-voltage, high-power equipment is simulated while the controllers under test are interfaced with the simulator and "tricked" into "thinking" that they are controlling actual power hardware. The interfaced controllers receive signals such as voltage and current measurements, execute their control algorithms, and feed signals for their control actions back into the simulator. By having all the high-power, high-voltage, equipment software-modeled, C-HIL enables wide test coverage of edge conditions such as faults, allowing non-destructive tests.

HIL involves the use of a real time simulator, such as the commercially available systems supplied by RTDS, OPAL-RT, Hypersim, and others. The advantages of this approach are to allow: (a) the reproduction of the distribution system operation in real time, with the possibility of synchronizing with an existing power system, if data from this system if available in real time; (b) the inclusion of real hardware components, in particular a hardware implementation of the microgrid controller, allowing HIL implementation and testing; (c) the use of models of components and systems with the appropriate degree of complexity needed to reproduce the microgrid environment, particularly for the non-critical components. Reducing the complexity of the models allows the reduction in the time needed to execute these models and representing the components as models reduces the amount of hardware needed. This approach is more expensive than the all-software approach, but significantly increases the level of confidence in the results and may reduce the validation requirements and time.

\section{B. Power HIL}

Power-HIL also uses a real-time simulation of a power system, but combines the simulation with actual fullvoltage, full-power hardware, Fig. 7. It employs, a highbandwidth power amplifier interfaced with the real-time simulator, which creates the voltage profiles required for the power equipment, for example a DER power electronic interface, under test, with profiles determined by the simulation. Power-HIL is typically used to test one component at full voltage and full power to evaluate its performance or to perform model validation and parameter identification of the DER. This Power-HIL testbed recreates the power system conditions that the equipment being tested would see on an actual power grid.

While Power-HIL typically involves testing of individual power components, it can also be employed for limited system integration and testing. The test fidelity for the equipment under test, interfaced with the simulation platform, will be high, but P-HIL does not offer a significant advantage in terms of fidelity over C-HIL for testing MGCS at system level, especially when using validated or manufacturer specified equipment models in the real-time simulator. Moreover, test coverage could be limited by the response bandwidth of the Power-HIL interface between real world equipment and the real-time simulator, and its power ratings. The range of tests may therefore be limited.

\section{Reduced scale test bed}

Subscale testbeds provide a scaled-down version of the microgrid power and control system to be deployed. The test equipment may operate at reduced voltages and power. Some organizations have developed power benchtop-level distribution testbeds operating at touch-safe voltages. Alternatively, the full system may be run with its final equipment at reduced voltage and power.

Depending on the scale factor and the level of voltage and power of the scaled-down system compared to the fullscale system, the subscale test bed can achieve a high test fidelity. However, the type of technology between scaledown equipment and final deployment should be taken into account appropriately. For example, most small power converters are single-phase and operate fundamentally differently than larger three-phase power converters. Controllers for smaller equipment may also operate differently, particularly in their grid support functionality, than controllers designed for larger ones. Since the subscale testbed offers a higher test fidelity, some microgrid owners, utility companies are interested in microgrid demonstration projects and testing of the control system in a scaled down version before large scale projects are implemented. 


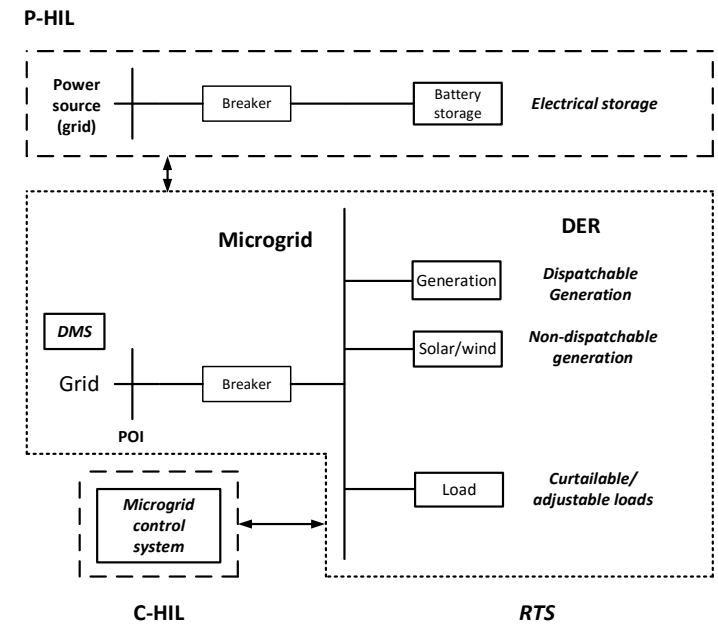

Fig. 7. P-HIL and C-HIL implementation block diagram - example.

\section{Full scale field testing}

Some utilities and equipment vendors have developed real size test systems, including substations and test feeders, into which they integrate new equipment for evaluation. These test installations may be operational customerserving facilities, or may simply serve dummy loads. When a full electric power system is populated with the components that are planned for deployment, this provides the highest level of test fidelity. Test coverage may be limited and component integration may be slowed, however, by concerns about operator and equipment safety. This is particularly a concern when testing under faults, overload conditions, and using newly developed software. Full-power system testbeds are among the most expensive to implement.

\section{E. Pure software simulation (off-line or real time)}

Software simulations are the traditional approaches in the study of power systems. They are usually performed under non-real-time conditions. Real Time Simulators (RTS) can be used for full software simulation or faster-than-real-time simulation. For most of the control system dispatch scenario evaluation, steady-state, or quasi steady state simulations may be sufficient. Simulations assessing the dynamics of the equipment within the power system may use generic, field validated or manufacturer specified models to allow designing the power and control systems and characterizing the desired performance. The fidelity of software simulation is related to the availability of validated models or equipment manufacturer supplied models. Software simulations enable an evaluation for a large and full range of test conditions and contingencies. Commercial software simulation packages, of the EMTP type, offer a wide range of models to simulate lines, transformers, DER and even some of the control and protection functions.

While the pure simulation approach may be used by microgrid controller developers in early stages of product development, the challenge is that the microgrid control system needs to be adequately reproduced in the simulation software. It can be supplied by the vendor.

\section{Conclusion}

This paper demonstrates that despite the wide range of implementations of microgrids and associated control functions, it is possible to define a generic control system, with a minimum of two core functions, that meets the requirements for a microgrid as per an agreed upon definition. It describes the microgrid control system standardization approach as carried out by the IEEE Standards Association working groups. It discusses the testing requirements and approaches, including software and hardware approaches.

\section{Acknowledgment}

This paper summarizes the work done under IEEE Standards Association Project Authorization Requests P2030.7 and P2030.8, approved by the IEEE Power and Energy Society Transmission and Distribution Committee and its Distributed Energy Resources Working Group. The work was carried out by the two working groups set up to write standards. Many stakeholders from different entities, including equipment manufacturers and vendors, government and research laboratories, and utilities have attended the meetings of the working groups over the twoyear duration of their respective mandate. The contributions of these stakeholders is duly acknowledged.

\section{References}

[1] NEMA MGRD 1-2016, Powering Microgrids for the 21stCentury Electrical System, August 2016.

[2] NYSERDA Report 14-36, Microgrids for Critical Facility Resiliency in New York State, Final Report, Dec. 2014.

[3] SAND2014-1535, The Advanced Microgrid - Integration and Interoperability, Bower, W. et al., Sandia National Laboratories, March 2014.

[4] DOE-FOA-997, Microgrid Research Development, and System Design, Funding Opportunity Announcement, National Energy Technology Laboratory, Jan. 2014.

[5] NEDO, Smart Grid EMS Functions in the Los Alamos Smart Grid Demonstrations, Hayashi, H., May 2013.

[6] California Public Utilities Commission, Microgrids: A Regulatory Perspective, Erickson, D., Policy \& Planning Division, April 14, 2014.

[7] N. Hatziargyriou ed., Microgrids: Architectures and Control, Wiley-IEEE Press, March 2014.

[8] C.A. Canizares, et al, "Trends in Microgrid Control", IEEE Task Force on Microgrid Control, IEEE Transactions on Smart Grid, July 2014.

[9] IEEE Std $1547^{\mathrm{TM}}$, IEEE Standard for Interconnecting Distributed Resources with Electric Power Systems.

[10] IEEE Std $1547 \mathrm{a}^{\mathrm{TM}_{-}}$2014, IEEE Standard for Interconnecting Distributed Resources with Electric Power Systems - Amendment 1.

[11] IEEE Std 1547.1 $1^{\mathrm{TM}}-2005$, Standard for Conformance Test Procedures for Equipment Interconnecting Distributed Resources with Electric Power Systems.

[12] IEEE Std 1547.2TM, Application Guide for IEEE 1547 Standard for Interconnecting Distributed Resources with Electric Power Systems. 